\title{
BMJ Open Changing contribution of area-level deprivation to total variance in age at death: a population-based decomposition analysis
}

\author{
Rosie Seaman, ${ }^{1}{ }^{1}$ Tim Riffe, ${ }^{1}$ Hal Caswell ${ }^{2}$
}

To cite: Seaman R, Riffe T, Caswell $\mathrm{H}$. Changing contribution of area-level deprivation to total variance in age at death: a populationbased decomposition analysis. BMJ Open 2019;9:e024952. doi:10.1136/ bmjopen-2018-024952

- Prepublication history and additional material for this paper are available online. To view these files, please visit the journal online (http://dx.doi org/10.1136/bmjopen-2018024952).

Received 26 June 2018 Revised 24 January 2019 Accepted 13 February 2019

Check for updates

(c) Author(s) (or their employer(s)) 2019. Re-use permitted under CC BY-NC. No commercial re-use. See rights and permissions. Published by BMJ.

${ }^{1}$ Max-Planck-Institut fur Demografische Forschung, Rostock, Germany

${ }^{2}$ Faculty of Science, University of Amsterdam, Amsterdam, The Netherlands

Correspondence to

Dr Rosie Seaman;

seaman@demogr.mpg.de

\section{ABSTRACT}

Objectives Two processes generate total variance in age at death: heterogeneity (between-group variance) and individual stochasticity (within-group variance). Limited research has evaluated how these two components have changed over time. We quantify the degree to which arealevel deprivation contributed to total variance in age at death in Scotland between 1981 and 2011.

Design Full population and mortality data for Scotland were obtained and matched with the Carstairs score, a standardised z-score calculated for each part-postcode sector that measures relative area-level deprivation. A z-score above zero indicates that the part-postcode sector experienced higher deprivation than the national average. A z-score below zero indicates lower deprivation. From the aggregated data we constructed 40 lifetables, one for each deprivation quintile in 1981, 1991, 2001 and 2011 stratified by sex.

Primary outcome measures Total variance in age at death and the proportion explained by area-level deprivation heterogeneity (between-group variance). Results The most deprived areas experienced stagnating or slightly increasing variance in age at death. The least deprived areas experienced decreasing variance. For males, the most deprived quintile life expectancy was between $7 \%$ and $11 \%$ lower and the SD is between $6 \%$ and $25 \%$ higher than the least deprived. This suggests that the effect of deprivation on the SD of longevity is comparable to its effect on life expectancy. Decomposition analysis revealed that contributions from between-group variance doubled between 1981 and 2011 but at most only explained $4 \%$ of total variance.

Conclusions This study adds to the emerging body of literature demonstrating that socio-economic groups have experienced diverging trends in variance in age at death. The contribution from area-level deprivation to total variance in age at death, which we were able to capture, has doubled since 1981. Area-level deprivation may play an increasingly important role in mortality inequalities.

\section{BACKGROUND}

The relationship between socio-economic inequality and mortality is traditionally based on life expectancy comparisons. The most deprived populations experience the lowest average age at death, and the least deprived
Strengths and limitations of this study

An advantage of this study is that it uses an indicator of area-level deprivation that is applicable to the entire population; existing studies of variation in age at death have used occupation or education as stratification measures which require left age truncation.

- The study uses a validated measure of area-level deprivation, specifically constructed for studying health inequalities and that covers three decades of population data.

- The Scottish Index of Multiple Deprivation (SIMD) is an alternative measure but it covers a far shorted period, and it includes an indicator of health which is theoretically problematic.

- We carry out robustness checks comparing the extreme deprivation quintiles of the Carstairs score only and by repeating our analyses using two variants of the SIMD.

populations experience the highest. ${ }^{1}$ Studies have further demonstrated that the most deprived populations also demonstrate the highest level of variation in age at death. Variation measured using e† was 8.5 years for the least income deprived group, compared with 12 years for the most income deprived group, in Denmark in 2014. ${ }^{2}$ Similarly, when measuring occupation in Finland the upper non-manual class variation was below 9 years while the manual class experience above 11 years variation in $2010 .^{3}$ Estimates for the USA and Spain using education to measure socio-economic inequality showed similar gaps in variation in age at death. ${ }^{45}$ Higher variation in age at death implies greater uncertainty, leading to the notion that the social patterning of these two distinct dimensions of mortality should be considered as a double burden of inequality.

Despite the growing body of evidence documenting the double burden of mortality inequality, variation in age at death is not yet routinely measured alongside life expectancy. 
This is important for evaluating the extent to which increases in average population health have been achieved alongside decreases in mortality inequality. ${ }^{67}$ Insights into drivers of total variation in age at death can be gained by quantifying two underlying processes: heterogeneity (between-group variance) and individual stochasticity (within-group variance). ${ }^{89}$

Within-group variance is due to differences among individuals in the outcome of stochastic demographic processes. Any quantity calculated from any lifetable (or a Markov matrix model) assumes that every individual, given its age or other state, is subject to the same set of mortality rates. Therefore, any variance in calculated age at death can be interpreted as the result of individual stochasticity. However, empirically measured within-group variance may also reflect unaccounted for subgroup heterogeneity. For example, males and females have different sets of mortality rates. Depending on the variance implied by each life table, a mixture of the two sexes may display larger or smaller variance than that of life table for only one of the two sexes (depending on which sex). Aggregating a lifetable over both sexes increases within-group variance due to induced between-group heterogeneity, even if males and females have identical within-group variance. ${ }^{10}$ This is a type of heterogeneity that we can typically account for by sex stratification, but the lifetable may contain other sources of heterogeneity that we cannot account for, either because we do not yet know, or because the information simply is not available. For example, we cannot always stratify our lifetables on all characteristics that are hypothesised to be important for mortality such as individual frailty, marital, employment, or diabetes status: characteristics of a population that are likely to account for some of the within-group component.

Between-group variance arises when individuals at the same age are subject to different mortality rates, which may be due to exposures to different social, economic, or environmental contexts. ${ }^{11}$ The contribution of educational inequalities (the between-group component) to the variability in age at death (measured by Theil's index of inequality rather than the variance) for 11 individual European countries has previously been estimated. ${ }^{12}$ For males in Sweden the between-group component accounted for $1.7 \%$ of the total variance in age at death but for males in the Czech Republic it accounted for $10.9 \%$ of total variance in age at death. The betweengroup component was higher in the Czech Republic because the age distributions of death, stratified by education, are more disparate than in Sweden. van Raalte et $a l^{12}$ used data aggregated over 1990-2000, such that time trends for the between-group component could not be assessed. It is also recognised that education may be a problematic socio-economic measure for studying trends in the between-group component due to changes in educational composition and the meaning of educational attainment. ${ }^{12} 13$ A further limitation when stratifying data by education is that researchers may need to left-truncate data at some age that may not be consistent across time or across comparison countries because education is an acquired characteristic.

Area-level measures of deprivation can be considered an alternative approach that can overcome this limitation: they are applicable to the whole population regardless of age. ${ }^{14} 15$ In empirical analyses, area-level measures of relative deprivation tend to be weighted into population quantiles. This gives a consistent interpretation over time; although geographical boundaries and absolute levels of poverty in a country may change, there is a notional most deprived fraction of the population that can be compared with the notional least deprived fraction of roughly the same size. In this paper, we are concerned with the effects of socio-economic inequality on longevity. We use an area-level measure of relative deprivation to understand the influence contextual deprivation may have on the risk of death. ${ }^{16-18}$

Inequalities in mortality and contrasting area-level deprivation are not unique to Scotland. However, it is a country of interest within the literature for at least three reasons. First, it experienced increasing mortality inequalities following the 1980s. ${ }^{19}{ }^{20}$ Second, it was shown to have the slowest improvements in life expectancy ${ }^{21}$ as well as the longest, sustained stagnating variance in age at death trend in Western Europe. ${ }^{22}$ Third, it demonstrated higher variance at shared levels of life expectancy when compared with England and Wales, despite arriving at the shared levels of life expectancy up to 10 years later. ${ }^{23} \mathrm{We}$ add to the existing knowledge about drivers of mortality inequalities by quantifying changes to the between-group and within-group components of total variance in age at death using an area-level measure of deprivation for stratification. We report results for four time points over three decades, and include ages $0-85$.

\section{DATA AND METHODS}

We used individual level mortality data and area-level population estimates that were obtained via a commissioned request from National Records of Scotland, ${ }^{24}$ alongside the Carstairs score of area-level deprivation that is freely available to access online. ${ }^{25}$ The data used for this study do not relate to individual patients.

Population data obtained were for each part-postcode sector in Scotland at each census year (1981, 1991, 2001 and 2011) and were stratified by single year of age and sex. Census years are considered to be the most robust population estimates available for the whole population of Scotland (ca 5 million persons), and these are used in subsequent mid-year population estimates.

Mortality data included all individual deaths that occurred in Scotland in the years centred on each census year. Each individual death record contains geographical information on place of usual residence (NRS policy for assigning geography to each death-deaths of Scottish residents that occur in Scotland are assigned to their place of normal residence, deaths of non-Scottish 
residents that occur in Scotland are assigned a geography based on their place of death, deaths of Scottish residents that occur outside of Scotland are not included.) ${ }^{26}$ that we used to match to population estimates and the Carstairs score. Deaths had to be excluded if they did not contain sufficient information on sex, age or postcode sector. These missing deaths represented a very small proportion $(<0.2 \%)$ of the total number of deaths included in the study, and it is unlikely that they would have impacted the substantive results that have been reported.

\section{Area-level deprivation}

The Carstairs score is a standardised z-score for each part-postcode sector that is derived from four individual-level census variables: overcrowding, male unemployment, low social class and car ownership. There are around 1012 part-postcode sectors in Scotland at each census year, with an average population size of 5000 individuals. Each part-postcode sector can be assigned to a quintile. The quintiles used in this study are population-weighted quintiles meaning that each quintile contains approximately $20 \%$ of individuals in the population. We chose to report results for quintiles of deprivation as they are the preferred analytical grouping for routine reporting of health measures in Scotland. ${ }^{27}$

The part-postcode sectors assigned to each quintile can change over time. We investigated the changing composition of each quintile over time by comparing the quintile of each part-postcode sector in each year with the modal
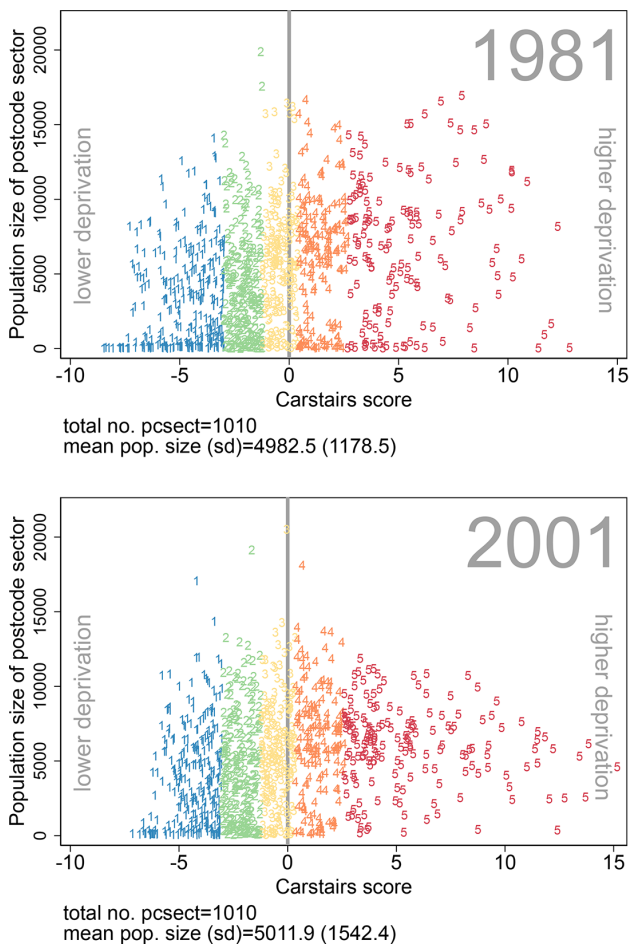

quintile of each part-postcode sector over the study period. Due to the data formatting, we had to limit this investigation to those postcode sectors that were never split between two Local Government District (1981 and 1991) or Council Area boundaries (2001 and 2011). This was 777 part-postcode sectors in 1981 and 874 part-postcode sectors in 2011. At least $75 \%$ of these part-postcode sectors were assigned to the same quintile as the modal quintile. Of the part-postcode sectors that differed from the modal quintile between $94 \%$ and $99 \%$ of them only moved to the neighbouring quintile. This demonstrates the relative consistency in the quintile composition over the study period.

The Carstairs score reflects the material resources that provide the means to access the goods, services, amenities and physical environment seen as expected in society. Since the score is centred on the population mean (grey vertical line in figure 1), it captures relative deprivation at the contextual level. ${ }^{18}$ In 2011, for example, scores ranged from -7.53 to 13.24 and were centred on zero, with higher scores indicating relatively higher deprivation than the national level. Figure 1 plots each part-postcode sector, which was included in our full analysis, against its Carstairs score and its population size at each census year.

\section{Life table construction and variance decomposition}

Deaths and census population denominators were used to construct complete lifetables for each deprivation quintile, centred on each census year, for males and females
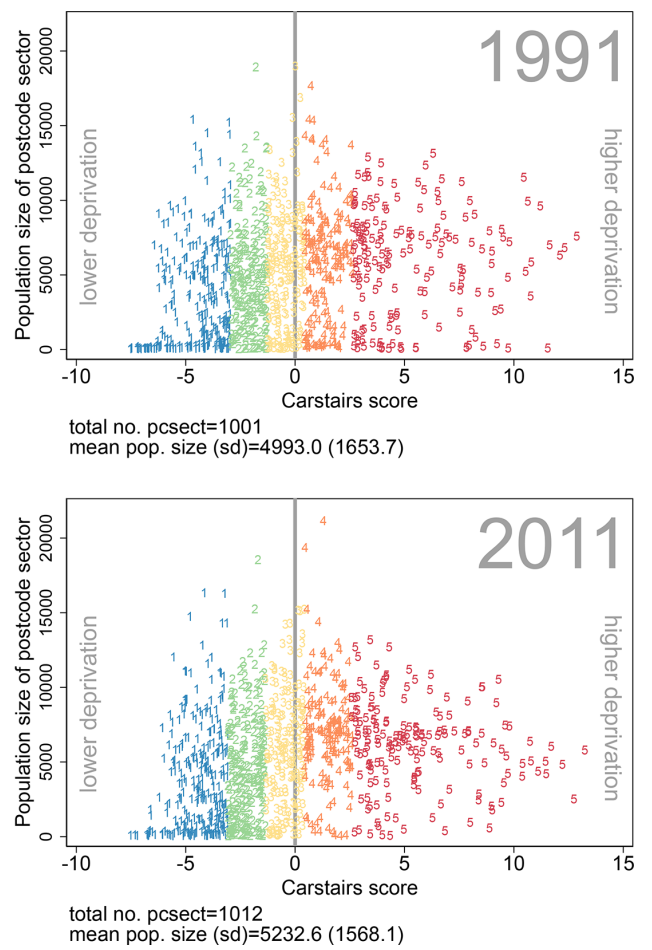

Figure 1 Carstairs score and population size for each part-postcode sector (pcsect) grouped by population weighted quintile at each census year. Each marker represents a single part-postcode sector. The numbers 1-5 show the quintile that each partpost code sector was assigned to. The grey 0 line is the national level of deprivation. Negative values on the $x$-axis indicate that the part-postcode sector experienced lower deprivation than the national level. Positive values on the $\mathrm{x}$-axis indicate higher deprivation than the national level. 
separately. The Human Mortality Database Methods Protocol was used to extrapolate age specific mortality rates from ages 85 to $110+{ }^{28}$ (Specifically, we apply equations (53) and (54) from the HMD protocol V.6, modified to use information from ages $75+$ rather than $80+$.)

From the complete lifetables we compute remaining life expectancy and the conditional variance of the remaining lifespan distribution for each age. A number of highly correlated indices can be used to measure variation in age at death. ${ }^{29}$ We use lifetable variance for two reasons. First, variance is one of only a few measures of variation that are additively decomposable into the between-group and within-group components. ${ }^{89}$ Second, we can transform variance into $\mathrm{SD}$, a common measure of the variability applied to the distribution of age at death, ${ }^{29}$ which allows for results to be interpreted intuitively in year units.

The contribution of area-level deprivation to the total variance in lifespan can be identified by carrying out a 'between-within decomposition' of variance. In this decomposition, the between-group component and the within-group component sum to the total variance (as calculated from the combined quintile-specific lifetables). Total variance is decomposed into two additive components representing the variance among individuals within quintiles, who by definition experience the same rates (within-group) and the variance due to quintile differences (between-group). Within-group variance is calculated as the weighted average of quintile-specific lifetable variances, with weights given by the proportion of the population in each quintile. The between-group variance is the weighted variance of the quintile means, with weights again given by the population composition. These calculations are conceptually identical to the calculation of variances within and among treatments in analysis of variance. We describe these steps in detail in online supplementary appendix 1 including the calculations of life expectancy, variance and variance decomposition. In addition, we provide all $\mathrm{R}$ scripts used for our analyses via the Open Science Framework (DOI 10.17605/OSF.IO/ Q4ZEJ).

\section{Sensitivity of results}

Our main results report life expectancy and SD at birth. To gauge how conservative our results were we carried out four sensitivity checks. First, we repeated the analysis using deciles of deprivation, each representing $10 \%$ of the population. The conclusions were the same for males and for females. The increase in the between-group component over time was slightly larger in magnitude when using deciles due to disaggregation of data, especially in the decile extremes.

Second, we examined how large the between-group component was when comparing the least deprived and most deprived quintiles only. The level of total variance, in this scenario, was higher but the patterns over time were unchanged. The between-group component was highest for males in 2001 accounting for 8\%: the between-group component was highest in 2011 for females accounting for $4 \%$.

Third, we estimated life expectancy trends and variation trends using two variants of the Scottish Index of Multiple Deprivation (SIMD): (1) the full SIMD weighted for all seven domains and (2) the income domain only of the SIMD (see also Discussion section). The trends are compared against ONS life expectancy estimates $^{31}$ in online supplementary appendix 2 . The direction of the trends is largely consistent, but levels are different. Part of the explanation comes from the fact that the Carstairs score and the SIMD are derived for different geographies. The SIMD was calculated for 6505 datazones in Scotland in 2011 compared with the Carstairs Score that was estimated for 1012 part-postcode sectors in 2011.

In 2011 only, the Carstairs score was calculated for datazones allowing for a fourth check. For this year we calculated life expectancy at birth and SD at birth (online supplementary appendix 3) and repeated the decomposition analysis (online supplementary appendix 4) for both variants of the SIMD and the Carstairs score for datazones. The amount of total variation explained by between-group variation is higher using the three alternative approaches that were all calculated for datazones. This reveals that the modifiable areal-unit problem is an important reason for the difference in magnitude between the Carstairs and SIMD. ${ }^{32}$ Our results reported using the Carstairs Score for part-postcode sectors can therefore be considered to be relatively conservative. Although the SIMD is the official deprivation measure used by the Scottish Government, we chose to report results for the Carstairs Score for part-postcode sectors because it is the recommended approach for examining longterm trends in area-level deprivation in Scotland. ${ }^{27}$

\section{RESULTS}

Figure 2 shows life expectancy and SD at birth, for each deprivation quintile and the total population estimate at each census year.

The most deprived quintile experienced the lowest life expectancy and the highest SD in age at death in each year. For males there was an increase in variation in age at death between 1991 and 2001. Although there was some improvement between 2001 and 2011, variation in age at death was very similar to the level experienced 30 years earlier.

Females from the most deprived quintile have experienced decreasing variation in age at death (decreasing SD) but the decrease was greater for the least deprived.

The effect of deprivation on the SD of longevity was comparable to, or even bigger than, its effect on life expectancy. For males in the most deprived quintile life expectancy was between $7 \%$ and $11 \%$ lower than the least deprived quintile, whereas the SD for the most deprived quintile was between $6 \%$ and $25 \%$ higher than variance 

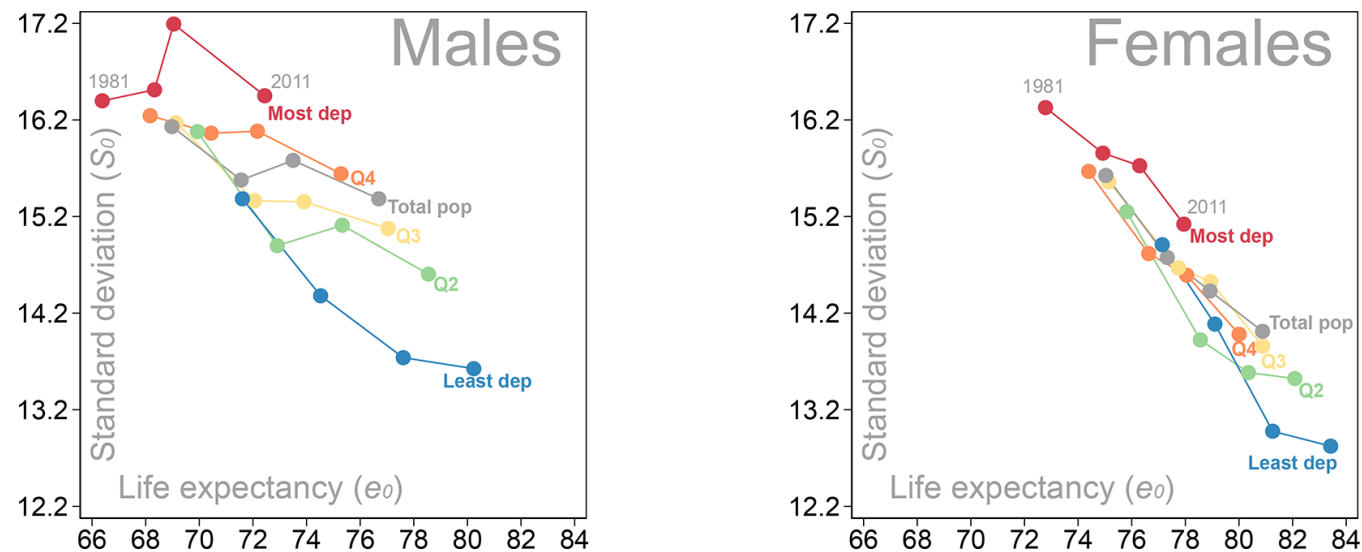

Figure 2 Relationship between Standard Deviation and life expectancy trend for each Carstairs deprivation quintile compared with the total population trend.

in the least deprived quintile. For females, life expectancy was lower by between $5 \%$ and $7 \%$ for the most deprived compared with the least deprived, and the SD was higher by between $9 \%$ and $21 \%$.

Figure 3 shows, for males and females, the proportion of the total variance of remaining longevity in Scotland due to differences between area-level deprivation quintiles as a function of age and time.

Despite the clear effect of socio-economic deprivation on variation trends, the deprivation differences contribute only a small fraction to the total variance in longevity (less than 4\%). However, the between-group component doubled between 1981 and 2011 for both males and females. This is the case for most cut-off ages but not across older ages where the between-group component was relatively constant over the study period. As a reference for the size of the between-group proportion and the increase, if we were to stratify by sex only, the between-sex component halved from 4\% in 1981 to roughly $2 \%$ in 2011 at most cut-off ages (see online supplementary appendix 5). This puts the between-sex and between-deprivation proportions on a similar scale.

For males the proportion of variance explained by between-group differences was lowest in 1981 and highest in 2001. By 2011 the proportion of between-group variance had decreased slightly but was still more than double that of 1981.

For females the proportion of variation explained by between-group variance was lowest in 1981 and highest in 2011. The crossover observed for males between 2001 and 2011 did not occur for females; instead the proportion explained by between-group variance consistently increased, doubling from 1981 to 2011.

For both sexes, the age pattern of the proportion of variance due to between-group differences was concave. The maximum between-group differences were found between ages 25 and 40 and the minimum betweengroup differences were found at older ages. This can be interpreted as variance in age at death being more dependent on area-level deprivation in young adulthood than in older ages. Considering that this analysis of arealevel deprivation has not previously been carried out for other populations, we do not know if this age pattern is a common feature of the relationship between area-level deprivation and mortality in developed countries or unique to Scotland. However, the maximum age in every census year is inclusive of the most common ages where alternative socio-economic measures may be forced to left truncate data.

\section{Proportion of between-group variance at each census year}
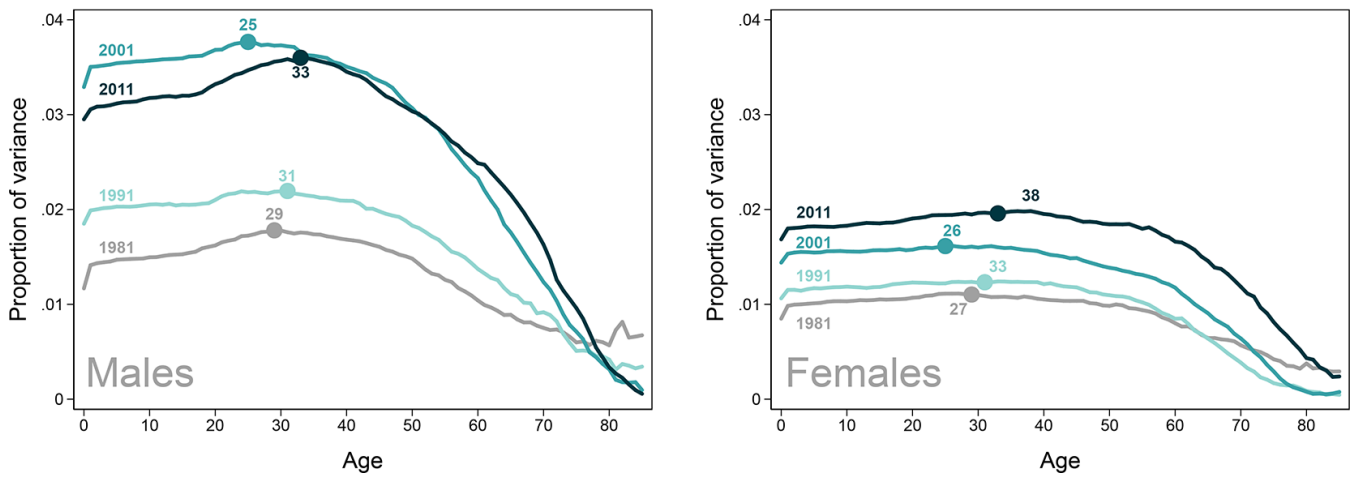

Figure 3 Proportion of variance due to differences between Carstairs deprivation quintiles. Age plotted is the age where the proportion of variance due to between-group inequality is highest in that year. 


\section{DISCUSSION AND CONCLUSION}

\section{Summary of main findings}

Deprivation differences in age at death patterns were evident at all census years when measuring socio-economic inequality at the area-level. The difference between deprivation groups was larger for males than for females. Males from the most deprived quintile experienced increasing variation in age at death between 1991 and 2001, followed by a decrease, such that the level of variation in 2011 was the same as that experienced 30 years earlier. The proportion of variance in age at death explained by the betweengroup component is very small, but was higher in 2011 than in 1981 for both males and females. The increase in the between-group component is driven by the fact that areas with relatively higher deprivation have experienced stagnating variation in age at death while areas with lower deprivation have continued to decrease variation. The between-group component is highest near age 30 , an age commonly used for left truncation in other studies that stratify lifetables by occupational groups or educational attainment.

\section{Relationship with existing literature}

Our study is the first to use an area-level measure of deprivation to demonstrate that those living in the most deprived areas can expect to live the shortest lives and experience the greatest variation in age at death. This finding is consistent with international evidence using income data for Denmark, ${ }^{2}$ occupational data for Finland ${ }^{3}$ and education data for the $\mathrm{USA}^{4}$ and Spain. ${ }^{5}$ If we consider steep socio-economic gradients in the uncertainty of longevity to be bad, then this consistent finding across contexts and across socio-economic measures represents a double burden of mortality inequality. Monitoring this double burden is important for informing public health policies about the extent to which improvements in the average health of populations have been achieved alongside reductions in inequality.

The small contribution of socio-economic differences to variance in age at death is in line with the findings of van Raalte et $a l^{12}$ (although they used a different metric), with analyses of Caswell et al, ${ }^{33}$ and with an analysis of variance contributed by heterogeneous frailty carried out by Hartemink et al. ${ }^{11}$ This does not mean that socio-economic inequality should be interpreted as having no effect on individuals; it reflects the high degree of stochasticity in this particular demographic outcome. Age at death is the result of a repeated series of mortality hazards experienced across the individual's life; such a process can be expected to produce a high variance due to individual stochasticity. The challenge is to extract the indicators of socio-economic inequality from the noise due to stochasticity.

Our results are important for highlighting the changing nature of age at death determinants; however, they are unable to identify the reason why the proportion explained by between-group variance increased. One interpretation of the results could be health selection effects either in terms of ill health causing downward mobility or in terms of meritocracy increasing upward mobility for individuals with favourable health characteristics. If either of these mechanisms were active then socio-economic groups would each be expected to become more homogeneous in terms of health, leading to diverging socio-economic differences in mortality outcomes. ${ }^{3}$

An alternative explanation is the well documented' polarisation' of deprivation, health and mortality that increased in the UK following the 1980s. ${ }^{3435}$ The timing of the increasing between-group component is in line with increases in relative inequality. A more recent event that could be of importance for increasing relative inequality and variation in age at death is the impact of the 2008 financial crisis. This topic requires further research attention. However, we believe our results contribute to the body of evidence showing the negative implications that relative deprivation can have on population level health and mortality. ${ }^{36}$ The results are particularly relevant for governments who are deciding how best to tackle mortality inequalities: whether to allocate resources to social policies that intervene at the contextual and arealevel versus social policies that intervene at the individual level. ${ }^{38-41}$ In addition to these theoretical contributions, our study demonstrates a number of empirical strengths.

\section{Strengths and limitations}

We used an empirically validated area-level measure of relative deprivation that has a consistent interpretation over a significant period of time, and that is recommended for long-term trend analyses. ${ }^{27}$ However, the measure is not without limitations. For example, the meaning of car ownership is fundamentally different for individuals in rural contexts compared with urban. Overcrowding may occur out of choice and for cultural reasons rather than simply being a marker of deprivation. ${ }^{42}$ Therefore it has been suggested that the Carstairs score may be an out-ofdate measure of socio-economic deprivation ${ }^{1620}$ : the relevance of the variables used for capturing the meaning of deprivation varies across contexts and over time. ${ }^{43}$

In response, it was demonstrated that the scores for each postcode sector at each census year are highly correlated despite changes to the formal definitions of the variables. This is interpreted as evidence that the underlying information the variables aim to capture is similar or that deprivation has remained stable over time. ${ }^{19}$

Alternative measures of area-level deprivation are available, and the official tool used by the Scottish Government is the SIMD. ${ }^{41}$ The SIMD includes 38 indicators from seven domains (employment, income, health, education, access to services, crime and housing). The SIMD was not suitable for the trend focus of this research, as it is only recommended for analysis using data beginning in $1996^{27}$ with population estimates for datazones only being available by single year of age from 2001 onwards.

A further theoretical limitation of the SIMD is that it includes indicators of health and mortality that would, to some extent, condition deprivation groups on the 
outcome. An alternative approach is to use the income domain only. ${ }^{44}$ The income domain is highly correlated with the full SIMD and is one of the most heavily weighted domains. We carried out sensitivity checks using both variants of the SIMD and for the Carstairs Score calculated for datazones. The results of these additional analyses showed our estimates from the Carstairs Score for part-postcode sectors to be the most conservative. However, our estimates of life expectancy ${ }^{31}$ and estimates variation in age at death are comparable and within the range of values reported from other populations and based on other measures. ${ }^{2-5} 45$

A further strength of our study is the data used: these are the most robust census population estimates and individual level mortality data covering the entire population of Scotland. This allowed the construction of complete lifetables with no left-truncation of age. However, it is important to acknowledge the reasons why studies interested in the social distribution mechanisms of adult mortality may consider restricting analysis to older ages. Smits and Monden ${ }^{7}$ suggest only looking at ages $15+$ because these are the ages where $80 \%$ of deaths in developed countries now occur. Looking only at adult mortality may better reflect the causes of death driving mortality change in more recent time periods: infectious disease and effective medical intervention historically reduced infant and childhood deaths rapidly, but reductions in adult mortality are influenced by more complex mechanisms that change slowly. ${ }^{746}$ Our results are important for this debate as they indicate that the age at which the difference in variation in age at death is greatest is around 30 years old. This provides some reassurance for studies that are forced to truncate younger age groups: the peak of variation in age at death (at least in developed countries) is likely to be captured.

\section{CONCLUSION}

Monitoring variance in age at death is complementary to the routine monitoring of life expectancy: monitoring both of these outcomes allows us to establish if average population mortality and mortality inequalities have been improving simultaneously. We find increasing contributions from area-level deprivation differences to total variance in age at death using population level data for Scotland. This type of trend analysis is important for understanding the changing nature of the social determinants of mortality inequalities in developed countries. More countries should begin to measure the betweengroup and within-group contributions to variance in age at death and monitor trends in order to understand the extent to which mortality is dependent on, and amenable to, relative area-level deprivation.

Collaborators Daniel Christoph Schneider1. Max-Planck-Institut fur Demografische ForschungRostock, DE 18057.
Contributors $\mathrm{RS}$ and $\mathrm{HC}$ came up with the initial research idea and research questions. RS and TR carried out the analyses jointly. All authors contributed to the writing and editing of the manuscript.

Funding Funding for this research comes from the European Research Council Starting Grant (716323), the European Union's Seventh Framework Programme (FP7/2007-2013) and the European Research Council Advanced Grant (322989).

Competing interests None declared.

Patient consent for publication Not required.

Ethics approval No ethical approval was required for this study. Data are for the full population of Scotland and can be independently requested.

Provenance and peer review Not commissioned; externally peer reviewed.

Data sharing statement All RScripts for analyses are available via the Open ScienceFramework (DOI 10.17605/OSF.IO/Q4ZEJ). Data required for the analyses can be independently downloaded from the relevant sources detailed in the Readme file or requested from NRS where necessary. The Carstairs score of deprivation data are available for download from the University of Glasgow. Population and mortality data for matching with the Carstairs score, and mortality data for datazones need to be independently requested from National Records of Scotland customer service team. The SIMD data are available for download from Information Services Division(ISD).Population estimates for datazones are available for download fromthe National Health Service open data source.

Open access This is an open access article distributed in accordance with the Creative Commons Attribution Non Commercial (CC BY-NC 4.0) license, which permits others to distribute, remix, adapt, build upon this work non-commercially, and license their derivative works on different terms, provided the original work is properly cited, appropriate credit is given, any changes made indicated, and the use is non-commercial. See: http://creativecommons.org/licenses/by-nc/4.0/.

\section{REFERENCES}

1. Marmot M, Allen J, Bell R, et al. WHO European review of social determinants of health and the health divide. Lancet 2012;380:1011-29.

2. Brønnum-Hansen $\mathrm{H}$. Socially disparate trends in lifespan variation: a trend study on income and mortality based on nationwide Danish register data. BMJ Open 2017;7:7.

3. van Raalte AA, Martikainen P, Myrskylä M. Lifespan variation by occupational class: compression or stagnation over time? Demography 2014;51:73-95.

4. Sasson I. Trends in life expectancy and lifespan variation by educational attainment: United States, 1990-2010. Demography 2016:1-25

5. Permanyer I, Spijker J, Blanes A, et al. Longevity and lifespan variation by educational attainment in Spain: 1960-2015. Demography 2018;55:2045-70.

6. Németh L. Life expectancy versus lifespan inequality: a smudge or a clear relationship? PLoS One 2017;12:e0185702.

7. Smits J, Monden C. Length of life inequality around the globe. Soc Sci Med 2009;68:1114-23.

8. Caswell H. A matrix approach to the statistics of longevity in heterogeneous frailty models. Demographic Research 2014;31:553-92.

9. Caswell H. Stage, age and individual stochasticity in demography. Oikos 2009;118:1763-82.

10. Andreev E, Lutz W, Scherbov S. Averaging life expectancy. IIASA Working Paper. Laxenburg, Austria, 1989.

11. Hartemink N, Missov TI, Caswell H. Stochasticity, heterogeneity, and variance in longevity in human populations. Theor Popul Biol 2017;114:107-16.

12. van Raalte $A A$, Kunst AE, Lundberg $O$, et al. The contribution of educational inequalities to lifespan variation. Popul Health Metr 2012;10:3.

13. Hendi AS. Trends in U.S. life expectancy gradients: the role of changing educational composition. Int J Epidemiol 2015;44:946-55.

14. Morgan O, Baker A. Measuring deprivation in England and Wales using 2001 Carstairs scores. Health Stat Q 2006:31.

15. Kearns A, Gibb K, Mackay D. Area deprivation in Scotland: a new assessment. Urban Stud 2000;37:1535-59.

16. Tunstall $H$, Mitchell $R$, Gibbs J, et al. Socio-demographic diversity and unexplained variation in death rates among the most deprived parliamentary constituencies in Britain. J Public Health 2012;34:296-304. 
17. Macintyre S, Ellaway A, Cummins S. Place effects on health: how can we conceptualise. operationalise and measure them? Social Science \& Medicine 2002:55.

18. Carstairs V, Morris R. Deprivation and mortality: an alternative to social class? Community Med 1989;11:210-9.

19. Leyland DR, McLoone P. Inequalities in mortality in Scotland, 1981 2001. occasional paper. Glasgow: Medical Research Council: Socia and Public Health Sciences Unit/Chief Scientist Office, 2007.

20. Schofield L, Walsh D, Munoz-Arroyo R, et al. Dying younger in Scotland: trends in mortality and deprivation relative to England and Wales, 1981-2011. Health Place 2016;40:106-15.

21. McCartney G, Walsh D, Whyte B, et al. Has Scotland always been the 'sick man' of Europe? An observational study from 1855 to 2006. Eur J Public Health 2012;22:756-60.

22. Seaman R, Leyland AH, Popham F. How have trends in lifespan variation changed since 1950? A comparative study of 17 Western European countries. Eur J Public Health 2016;26:360-2.

23. Seaman R, Leyland AH, Popham F. Increasing inequality in age of death at shared levels of life expectancy: a comparative study of Scotland and England and Wales. SSM Popul Health 2016;2:724-31.

24. National Records of Scotland. http://www.nrscotland.gov.uk. 2017.

25. Brown D, Allik M, Dundas R, et al. Carstairs Scores for Scottish Postcode Sectors, Datazones and Output Areas from the 2011 Census. Glasgow: Technical Report MRC/CSO Social and Public Health Sciences Unit, University of Glasgow, 2014.

26. General Register Office for Scotland. Vital events-deaths-background information: place of death and place of occurrence. 2016.

27. NHS Public Health and Intelligence. Deprivation guidance for analysts, National Services Scotland. 2017.

28. Wilmoth JR, Andreev K, Jdanov D, et al. Methods protocol for the human mortality database University of California, Berkeley, and Max Planck Institute for Demographic Research, Rostock. 2017.

29. van Raalte $A A$, Caswell $H$. Perturbation analysis of indices of lifespan variability. Demography 2013;50:1615-40.

30. Caswell H. Construction, analysis, and interpretation. Sunderland: Sinauer, 2001.

31. Office for National Statistics. Expectation of life, principal projection, Scotland. 2017.

32. Openshaw S. Ecological fallacies and the analysis of areal census data. Environment and Planning A: Economy and Space. 1984;16:17-31.
33. Caswell H, de Vries $\mathrm{C}$, Hartemink N, et al. Age $\times$ stage-classified demographic analysis: a comprehensive approach. Ecol Monogr 2018;88:560-84.

34. Shaw M, Gordon D, Dorling D, et al. Increasing mortality differentials by residential area level of poverty: Britain 1981-1997. Soc Sci Med 2000;51:151-3.

35. Mitchell R, Dorling D, Shaw M. In: Press TP, ed. Inequalities in life and death. What if Britain were more equal? Bristol: The Joseph Rowntree Foundation, 2000.

36. Marmot M, Wilkinson RG. Psychosocial and material pathways in the relation between income and health: a response to Lynch et al. BMJ 2001;322:1233-6.

37. Wilkinson RG, Pickett KE. The problems of relative deprivation: why some societies do better than others. Soc Sci Med 2007;65:1965-78.

38. Allik M, Brown D, Dundas R, et al. Developing a new small-area measure of deprivation using 2001 and 2011 census data from Scotland. Health Place 2016;39:122-30.

39. Diez Roux AV. Investigating neighborhood and area effects on health Am J Public Health 2001;91:1783-9.

40. Robert SA. Socioeconomic position and health: the independent contribution of community socioeconomic context. Annu Rev Sociol 1999;25:489-516.

41. The Scottish Government. Scottish index of multiple deprivation: ranks and domain ranks: Edinburgh National Statistics, 2016.

42. Fischbacher CM. Identifying "Deprived Individuals": Are There Better Alternatives to the Scottish Index of Multiple Deprivation (SIMD) for Socioeconomic Targeting in Individually Based Programmes Addressing Health Inequalities in Scotland. Edinburgh, UK: Scottish Public Health Organisation, 2014.

43. Norman P. Identifying change over time in small area socio-economic deprivation. App/ Spat Anal Policy 2010;3:107-38.

44. Leyland AH, Dundas R, McLoone P, et al. Cause-specific inequalities in mortality in Scotland: two decades of change. A population-based study. BMC Public Health 2007;7:172-84.

45. van Raalte AA, Kunst AE, Deboosere P, et al. More variation in lifespan in lower educated groups: evidence from 10 European countries. Int J Epidemiol 2011;40:1703-14.

46. Vallin J, Meslé F. Convergences and divergences in mortality: a new approach of health transition. Demographic Research 2004:11-44. 